\title{
Sources of Professional Knowledge for Academic Librarians
}

\author{
Ronald R. Powell
}

\begin{abstract}
More than three hundred ARL librarians were asked where they had acquired their professional knowledge and where they thought it would be best to acquire it. Respondents indicated that library school and on-the-job experience provided most of their professional education and training but suggested that they would prefer to acquire more of their knowledge from continuing education and staff development programs than is presently the case. Other sources of information investigated include nonlibrary science degree programs and internships. Earlier research on the amount and importance of university librarians' professional knowledge is summarized.
\end{abstract}

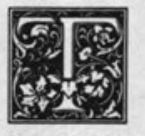

he demands being made of academic librarians are changing at a rapid rate. Librarians also need to be proficient in an everincreasing number of areas. What is not known with any certainty is exactly what skills are most important for librarians and where those skills are optimally acquired.

The knowledge, skills, and/or proficiencies needed by academic librarians have received considerable attention in the literature during the past several years. Articles and other reports have been authored by, among others, Millicent Abell, ${ }^{1}$ Toni Carbo Bearman, ${ }^{2}$ Patricia Battin, ${ }^{3}$ Sheila Creth and Faith Harders, ${ }^{4}$ the Association of Research Libraries (ARL), ${ }^{5}$ and JoseMarie Griffiths and Donald King. ${ }^{6}$ Yet there is still a need for more specific data on the proficiencies important to university librarians.

Ronald Powell and Sheila Creth conducted a study in 1985 designed to answer the following questions:
1. To what extent do librarians consider themselves knowledgeable in relevant areas?

2. To what extent are these areas of knowledge considered important for effective job performance?

3. Where do librarians tend to acquire their relevant knowledge?

4. Where do librarians think such knowledge is best acquired?

In order to gather necessary data to answer these questions, the researchers mailed questionnaires to a random sample of 539 ARL librarians with nine or fewer years of professional experience. Three hundred and forty-nine usable questionnaires were returned, representing about 65 percent of the sample. The questionnaires collected information about the librarians' current positions, job and educational experiences, sex, age, and knowledge bases. (Knowledge base denotes the proficiencies, skills, and information possessed by the participating librarians.)

Ronald R. Powell is Associate Professor at the School of Library and Informational Science, University of Missouri-Columbia, Columbia, Missouri 65211. The author wishes to thank Sheila D. Creth, Library Director, University of Iowa, who assisted in the research and co-authored an earlier report. This research was made possible by a Faculty/Librarian Cooperative Research grant provided by the Council on Library Resources. 
Initial analysis of the data focused on the professional knowledge of the respondents and the value they attached to specific knowledge bases. The results of that analysis were reported in the January 1986 issue of College \& Research Libraries. ${ }^{7}$ A brief summary of those findings follows.

\section{IMPORTANCE AND AMOUNT OF KNOWLEDGE}

For each of fifty-six knowledge bases, participants indicated how much of the knowledge they possessed and how important it was for their own job performance. The fifty-six knowledge bases are presented in table 1 (table 2 of the 1986 article) in order of their perceived importance (see the first two columns). The third and fourth columns of table 1 indicate how much knowledge was held by the respondents and the corresponding rankings. The importance of each knowledge base was measured on a five-point scale: one designates essential; two, very important; three, important; four, of little importance; and five, of no importance. The amount of each knowledge base possessed by respondents was measured on the following four-point scale: one, extensive; two, moderate; three, slight; and four, none.

In brief, an examination of the data in table 1 reveals that, generally, traditional knowledge areas tend to be ranked relatively high in importance by respondents. In fact, most of the top twenty areas fell into one of two categories: traditional core and management. ${ }^{8}$ Analysis of the data also indicated that several of the knowledge bases, especially less traditional ones, that were considered quite important did not receive correspondingly high rankings on the amount of the knowledge that the respondents reported they actually possessed.

The results of the analysis supported the authors' contention that not every important skill is being acquired in library school. But given that it is not possible for a student to learn in one or two years all that he or she will need throughout an entire professional career, we are left with the question of where the important skills can be most conveniently and effectively acquired. Consequently, data relating to where the respondents had acquired their professional knowledge and where they thought it would best be acquired were analyzed.

\section{KNOWLEDGE BASES: WHERE ACQUIRED?}

The following analysis, a summary of the data regarding where librarians acquire their knowledge and where they think it should be acquired, is limited to the knowledge bases ranked among the twenty most important and the top twenty in terms of amount held.

The majority of knowledge bases were acquired in library school and on the job (see table 2). (The number of "yes" responses can be greater than the number of respondents as many skills were acquired from more than one source.) Almost 29 percent of all "yes" responses represent library school and about 35 percent onthe-job experiences. Continuing education and staff development combined represented over 16 percent of the "yes" responses. Other degree programs, internships, and "other" accounted for the remaining 20 percent.

The skills reported as being most frequently acquired in library school were familiarity with and use of: bibliographic tools, general reference sources, reference interview, cataloging codes/rules, subject cataloging, subject classification, and the structure of subject literature. All of these bases represent traditional core areas of formal library education programs.

On the other hand, the proficiencies most frequently obtained on the job involved: oral communication skills, knowledge of specialized reference sources, decision-making ability, search strategy, planning, online searching, selection of materials, personnel management, library automation, structure of subject literature (tie), bibliographic instruction, and staff training and development. These skills are generally less traditional in nature than those more frequently acquired in library school. The two remaining proficiencies, writing skills and knowledge of a subject field, were most frequently gained in other degree programs. 
TABLE 1

RANKING AND MEAN SCORES FOR "IMPORTANCE" OF KNOWLEDGE BASE AND "AMOUNT" OF KNOWLEDGE

\begin{tabular}{|c|c|c|c|c|}
\hline Knowledge Base & \multicolumn{2}{|c|}{$\begin{array}{l}\text { Importance of Knowledge } \\
\text { Mean Score (1-5) }\end{array}$} & \multicolumn{2}{|c|}{$\begin{array}{l}\text { Amount of Knowledge } \\
\text { Mean Score (1-5) }\end{array}$} \\
\hline Bibliographic tools & 1.67 & 1.5 & 1.39 & 1 \\
\hline Oral communication skills & 1.67 & 1.5 & 1.76 & 8 \\
\hline Writing skills & 1.71 & 3 & 1.53 & 2 \\
\hline Specialized reference sources & 1.95 & 4 & 1.63 & 4 \\
\hline Decision making & 2.05 & 5 & 2.03 & 18 \\
\hline Search strategy & 2.07 & 6 & 1.58 & 3 \\
\hline Subject field & 2.10 & 7 & 1.64 & \\
\hline General reference sources & 2.17 & 8 & 1.64 & 5.5 \\
\hline Planning & 2.20 & 9 & 2.12 & 20 \\
\hline Online searching & 2.38 & 10 & 1.86 & 9 \\
\hline Reference interview & 2.39 & 11 & 1.70 & 7 \\
\hline Selection of materials & 2.41 & 12.5 & 1.87 & 10 \\
\hline Catalog codes/rules & 2.41 & 12.5 & 1.95 & 13.5 \\
\hline Personnel management & 2.42 & 14 & 2.23 & 27 \\
\hline Subject cataloging & 2.43 & 15.5 & 2.01 & 17 \\
\hline Library automation & 2.43 & 15.5 & 2.13 & 21 \\
\hline Subject classification & 2.48 & 17 & 1.94 & 12 \\
\hline Structure of subject literature & 2.53 & 18 & 1.99 & 16 \\
\hline Bibliographic/library instruction & 2.55 & 19 & 1.98 & 15 \\
\hline Staff training and development & 2.56 & 20 & 2.36 & 32 \\
\hline Filing & 2.59 & 21 & 1.92 & 11 \\
\hline Research methods & 2.68 & 22 & 1.95 & 13.5 \\
\hline Collection evaluation & 2.72 & 23 & 2.22 & 25 \\
\hline Nonbook formats & 2.78 & 24 & 2.22 & 25 \\
\hline Acquisitions procedures & 2.82 & 25 & 2.17 & 22 \\
\hline Teaching methods & 2.84 & 26 & 2.25 & 28 \\
\hline Higher education & 2.89 & 27 & 2.08 & 19 \\
\hline Computer software & 3.01 & 28 & 2.53 & 38 \\
\hline Budgeting & 3.02 & 29 & 2.57 & 40 \\
\hline Management theory & 3.04 & 30 & 2.45 & 36 \\
\hline Foreign language & 3.05 & 31 & 2.21 & 23 \\
\hline Collection weeding & 3.06 & 32 & 2.37 & 33 \\
\hline Resource sharing & 3.08 & 34 & 2.33 & 29 \\
\hline Program evaluation techniques & 3.08 & 34 & 2.79 & 49 \\
\hline Networks & 3.08 & 34 & 2.35 & 30.5 \\
\hline Space and work environment & 3.09 & 36 & 2.52 & 37 \\
\hline Publishing industry & 3.12 & 37 & 2.42 & 34 \\
\hline Cataloging of specíal materials & 3.18 & 38 & 2.62 & 45 \\
\hline Computer hardware & 3.20 & 39 & 2.62 & 45 \\
\hline Indexing & 3.23 & 40 & 2.35 & 30.5 \\
\hline Serials control & 3.25 & 41 & 2.63 & 47 \\
\hline Preservation/conservation & 3.28 & 42 & 2.62 & 45 \\
\hline Copyright & 3.29 & 43 & 2.44 & 35 \\
\hline Collection storage & 3.38 & 44 & 2.59 & 42 \\
\hline Approval plans & 3.39 & 45 & 2.56 & 39 \\
\hline Circulation services & 3.42 & 46 & 2.22 & 25 \\
\hline System analysis & 3.48 & 47 & 2.86 & 53 \\
\hline Circulation systems & 3.62 & 48 & 2.60 & 43 \\
\hline Cooperative acquisitions & 3.68 & 49 & 2.82 & 51 \\
\hline Security systems & 3.74 & 50 & 2.82 & 51 \\
\hline Commercial or external & & & & \\
\hline $\begin{array}{l}\text { cataloging services } \\
\text { Computer programming }\end{array}$ & $\begin{array}{l}3.76 \\
3.81\end{array}$ & 51 & 2.82 & $\begin{array}{l}51 \\
54\end{array}$ \\
\hline $\begin{array}{l}\text { Computer programming } \\
\text { History of books and printing }\end{array}$ & $\begin{array}{l}3.81 \\
3.87\end{array}$ & 52 & 2.99 & $\begin{array}{l}54 \\
41\end{array}$ \\
\hline $\begin{array}{l}\text { History of books and printing } \\
\text { Inferential statistics }\end{array}$ & $\begin{array}{l}3.87 \\
3.94\end{array}$ & $\begin{array}{l}53 \\
54\end{array}$ & $\begin{array}{l}2.58 \\
3.29\end{array}$ & $\begin{array}{l}41 \\
55\end{array}$ \\
\hline History of libraries & 4.16 & 55 & 2.67 & 48 \\
\hline Collective bargaining & 4.28 & 56 & 3.35 & 56 \\
\hline
\end{tabular}


TABLE 2

TWENTY MOST IMPORTANT KNOWLEDGE BASES

AND YES RESPONSES FOR WHERE ACQUIRED

\begin{tabular}{|c|c|c|c|c|c|c|c|}
\hline \multirow[b]{2}{*}{ Knowledge Base } & \multirow[b]{2}{*}{$\begin{array}{l}\text { Library } \\
\text { School }\end{array}$} & \multirow[b]{2}{*}{$\begin{array}{c}\text { Other } \\
\text { Degree } \\
\text { Programs }\end{array}$} & \multicolumn{3}{|c|}{ Knowledge Source } & \multirow[b]{2}{*}{ On-the-Job } & \multirow[b]{2}{*}{ Other } \\
\hline & & & Internship & $\begin{array}{l}\text { Continuing } \\
\text { Education } \\
\end{array}$ & $\begin{array}{c}\text { Staff } \\
\text { Development } \\
\end{array}$ & & \\
\hline $\begin{array}{l}\text { Bibliographic tools } \\
\text { Percent }\end{array}$ & $\begin{array}{l}299 \\
34.5\end{array}$ & $\begin{array}{l}37 \\
4.3\end{array}$ & $\begin{array}{l}57 \\
6.6\end{array}$ & $\begin{array}{l}79 \\
9.1\end{array}$ & $\begin{array}{l}52 \\
6.0\end{array}$ & $\begin{array}{l}289 \\
33.3\end{array}$ & $\begin{array}{ll}155 \\
\quad 6.3\end{array}$ \\
\hline Oral communication skills & 80 & 168 & 21 & 65 & 52 & 186 & 141 \\
\hline Percent & 11.2 & 23.6 & 2.9 & 9.1 & 7.3 & 26.1 & 19.8 \\
\hline Writing skills & 69 & 245 & 11 & 50 & 24 & 137 & 138 \\
\hline Percent & 10.2 & 36.4 & 1.6 & 7.4 & 3.6 & 20.3 & 20.5 \\
\hline Specialized reference sources & 241 & 56 & 44 & 63 & 46 & 269 & 30 \\
\hline Percent & 32.2 & 7.5 & 5.9 & 8.4 & 6.1 & 35.9 & 4.0 \\
\hline Decision making & 123 & 39 & 20 & 70 & 51 & 253 & 82 \\
\hline Percent & 19.3 & 6.1 & 3.1 & 11.0 & 8.0 & 39.7 & 12.9 \\
\hline Search strategy & 225 & 21 & 33 & 75 & 53 & 267 & 40 \\
\hline Percent & 31.5 & 2.9 & 4.6 & 10.5 & 7.4 & 37.4 & 5.6 \\
\hline Subject field & 63 & 222 & 25 & 101 & 25 & 185 & 69 \\
\hline Percent & 9.1 & 32.2 & 3.6 & 14.6 & 3.6 & 26.8 & 10.0 \\
\hline General reference sources & 311 & 29 & 44 & 48 & 41 & 260 & 43 \\
\hline Percent & 40.1 & 3.7 & 5.7 & 6.2 & 5.3 & 33.5 & 5.5 \\
\hline Planning & 161 & 34 & 16 & 75 & 59 & 256 & 71 \\
\hline Percent & 24.0 & 5.1 & 2.4 & 11.2 & 8.8 & 38.1 & 10.6 \\
\hline Online searching & 189 & 4 & 26 & 114 & 75 & 248 & 28 \\
\hline Percent & 27.6 & 0.6 & 3.8 & 16.7 & 11.0 & 36.3 & 4.1 \\
\hline Reference interview & 263 & 10 & 37 & 41 & 33 & 240 & 33 \\
\hline Percent & 68.5 & 2.6 & 9.6 & 10.7 & 8.6 & 62.5 & 8.6 \\
\hline Selection of materials & 213 & 30 & 28 & 42 & 19 & 269 & 44 \\
\hline Percent & 33.0 & 4.7 & 4.3 & 6.5 & 2.9 & 41.7 & 6.8 \\
\hline Catalog codes/rules & 298 & 5 & 31 & 49 & 41 & 235 & 38 \\
\hline Percent & 42.8 & 0.7 & 4.5 & 7.0 & 5.9 & 33.7 & 5.5 \\
\hline Personnel management & 141 & 32 & 12 & 76 & 78 & 247 & 65 \\
\hline Percent & 21.7 & 4.9 & 1.8 & 11.7 & 12.0 & 37.9 & 10.0 \\
\hline Subject cataloging & 258 & 4 & 27 & 29 & 24 & 206 & 34 \\
\hline Percent & 44.3 & 0.7 & 4.6 & 5.0 & 4.1 & 35.4 & 5.8 \\
\hline Library automation & 236 & 3 & 24 & 95 & 72 & 279 & 47 \\
\hline Percent & 31.2 & 0.4 & 3.2 & 12.6 & 9.5 & 36.9 & 6.2 \\
\hline Subject classification & 271 & 13 & 28 & 27 & 20 & 220 & 29 \\
\hline Percent & 44.6 & 2.1 & 4.6 & 4.4 & 3.3 & 36.2 & 4.8 \\
\hline Structure of subject literature & 209 & 62 & 27 & 48 & 22 & 209 & 25 \\
\hline Percent & 34.7 & 10.3 & 4.5 & 8.0 & 3.7 & 34.7 & 4.2 \\
\hline Bibliographic/library instruction & 160 & 20 & 19 & 73 & 52 & 257 & 44 \\
\hline Percent & 35.6 & 3.2 & 3.0 & 11.5 & 8.3 & 41.1 & 7.0 \\
\hline Staff training \& development & 71 & 21 & 9 & 70 & 90 & 238 & 49 \\
\hline Percent & 13.0 & 3.8 & 1.6 & 12.8 & 16.4 & 43.4 & 8.9 \\
\hline Totals & 3,881 & 1,055 & 539 & 1,290 & 929 & 4,750 & 1,105 \\
\hline Percent & 28.6 & 7.8 & 4.0 & 9.5 & 6.9 & 35.1 & 8.2 \\
\hline
\end{tabular}

Table 3 lists the twenty knowledge bases most highly ranked on the amount of the knowledge possessed by the survey respondents. There are only three skills in this table that were not included in table 2. Research methods, one of the additions, was reported as being most frequently acquired in library school. Knowledge of filing rules and higher education were most often obtained on the job.

In that the contents of the tables are almost the same, it is not surprising that the totals are very similar. The respondents indicated that library school was the source of knowledge almost 30 percent of the time, on-the-job experiences represented 34 percent of the sources, and continuing education and staff development activities accounted for over 14 percent of the affirmative responses.

\section{KNOWLEDGE BASES: WHERE BEST ACQUIRED?}

In contrast, the responses regarding 
TABLE 3

TWENTY TOP KNOWLEDGE BASES RANKED BY AMOUNT HELD AND YES RESPONSES FOR WHERE ACQUIRED

\begin{tabular}{|c|c|c|c|c|c|c|c|}
\hline \multirow[b]{2}{*}{ Knowledge Base } & \multirow[b]{2}{*}{$\begin{array}{l}\text { Library } \\
\text { School }\end{array}$} & \multirow[b]{2}{*}{$\begin{array}{c}\begin{array}{c}\text { Other } \\
\text { Degree } \\
\text { Programs }\end{array} \\
\end{array}$} & \multirow[b]{2}{*}{ Internship } & \multicolumn{2}{|c|}{ Knowledge Source } & \multirow[b]{2}{*}{ On-the-Job } & \multirow[b]{2}{*}{ Other } \\
\hline & & & & $\begin{array}{l}\text { Continuing } \\
\text { Education }\end{array}$ & $\begin{array}{c}\text { Staff } \\
\text { Development }\end{array}$ & & \\
\hline & 299 & ${ }_{43}^{37}$ & 57 & 79 & 52 & & 55 \\
\hline $\begin{array}{l}\text { Percent } \\
\text { Writing skills }\end{array}$ & 34.5 & $245^{4.3}$ & 6.6 & ${ }_{50}^{9.1}$ & $24^{6.0}$ & 33.3 & $\begin{array}{r}6.3 \\
138\end{array}$ \\
\hline & $\begin{array}{l}69 \\
10.2\end{array}$ & 36.4 & 1.6 & $\begin{array}{l}50 \\
7.4\end{array}$ & $\begin{array}{r}24 \\
3.6\end{array}$ & $\begin{array}{r}137 \\
20.3\end{array}$ & $\begin{array}{l}138 \\
20.5\end{array}$ \\
\hline Search strategy & 225 & 21 & 33 & 75 & 53 & 267 & $\begin{array}{l}20.3 \\
40\end{array}$ \\
\hline Percent & 31.5 & 2.9 & 4.6 & 10.5 & 7.4 & 37.4 & 5.6 \\
\hline Specialized reference sources & 241 & 56 & 44 & 63 & 46 & 269 & 30 \\
\hline Percent & 32.2 & 7.5 & 5.9 & 8.4 & 6.1 & 35.9 & 4.0 \\
\hline Subject field & 63 & 222 & 25 & 101 & 25 & 185 & 69 \\
\hline Percent & 9.1 & 32.2 & 3.6 & 14.6 & 3.6 & 26.8 & 10.0 \\
\hline General reference sources & 311 & 29 & 44 & 48 & 41 & 260 & 43 \\
\hline Percent & 40.1 & 3.7 & 5.7 & 6.2 & 5.3 & 33.5 & 5.5 \\
\hline Reference interview & 263 & 10 & 37 & 41 & 33 & 240 & 33 \\
\hline Percent & 68.5 & 2.6 & 9.6 & 10.7 & 8.6 & 62.5 & 8.6 \\
\hline Oral communication skills & 80 & 168 & 21 & 65 & 52 & 186 & 141 \\
\hline Percent & 11.2 & 23.6 & 2.9 & 9.1 & 7.3 & 26.1 & 19.8 \\
\hline Online searching & 189 & 4 & 26 & 114 & 75 & 248 & 28 \\
\hline Percent & 27.6 & 0.6 & 3.8 & 16.7 & 11.0 & 36.3 & 4.1 \\
\hline Selection of materials & 213 & 30 & 28 & 42 & 19 & 269 & 44 \\
\hline Percent & 33.0 & 4.7 & 4.3 & 6.5 & 2.9 & 41.7 & 6.8 \\
\hline Filing & 191 & 5 & 32 & 8 & 17 & 252 & 49 \\
\hline Percent & 34.5 & 0.9 & 5.8 & 1.4 & 3.1 & 45.5 & 8.8 \\
\hline Subject classification & 271 & 13 & 28 & 27 & 20 & 220 & 29 \\
\hline Percent & 44.6 & 2.1 & 4.6 & 4.4 & 3.3 & 36.2 & 4.8 \\
\hline Catalog codes/rules & 298 & 5 & 31 & 49 & 41 & 235 & 38 \\
\hline Percent & 42.8 & 0.7 & 4.5 & 7.0 & 5.9 & 33.7 & 5.5 \\
\hline Research methods & 214 & 165 & 15 & 56 & 20 & 143 & 46 \\
\hline Percent & 32.5 & 25.0 & 2.3 & 8.5 & 3.0 & 21.7 & 7.0 \\
\hline Bibliographic/library instruction & 160 & 20 & 19 & 73 & 52 & 257 & 44 \\
\hline Percent & 25.6 & 3.2 & 3.0 & 11.5 & 8.3 & 41.1 & 7.0 \\
\hline Structure of subject literature & 209 & 62 & 27 & 48 & 22 & 209 & 25 \\
\hline Percent & 34.7 & 10.3 & 4.5 & 8.0 & 3.7 & 34.7 & 4.2 \\
\hline Subject cataloging & 258 & 4 & 27 & 29 & 24 & 206 & 34 \\
\hline Percent & 44.3 & 0.7 & 4.6 & 5.0 & 4.1 & 35.4 & 5.8 \\
\hline Decision making & 123 & 39 & 20 & 70 & 51 & 253 & 82 \\
\hline Percen & 19.3 & 6.1 & 3.1 & 11.0 & 8.0 & 39.7 & 12.9 \\
\hline Higher education & 100 & 112 & 9 & 48 & 20 & 162 & 83 \\
\hline Percent & 18.7 & 21.0 & 1.7 & 9.0 & 3.7 & 30.3 & 15.5 \\
\hline Planning & 161 & 34 & 16 & 75 & 59 & 256 & 71 \\
\hline Percent & 24.0 & 5.1 & 2.4 & 11.2 & 8.8 & 38.1 & 10.6 \\
\hline Totals & 3,938 & 1,281 & 550 & 1,161 & 746 & 4,543 & 1,122 \\
\hline Percent & 29.5 & 9.6 & 4.1 & 8.7 & 5.6 & 34.1 & 8.4 \\
\hline
\end{tabular}

where the librarians believed the proficiencies would best be acquired produced some different patterns (see table 4). The participating librarians indicated nine proficiencies (of the twenty most important) that were best learned in library schools. Those were: bibliographic tools, search strategy, general reference sources, reference interview, selection of materials, cataloging codes/rules, subject cataloging, subject classification, and the structure of subject literature. Two of these areas of knowledge (search strategy and selection of materials) were not among the skills most frequently acquired in library school.

A more substantial difference was found in analyzing perceptions of the knowledge bases best acquired on the job. In fact, the workplace was seen as the optimal place to acquire only two knowledge bases: specialized reference sources and selection of materials (tie). In contrast, twelve knowledge bases were reported as having been most frequently obtained on the job. This finding seems to correspond with White and Paris' observation that 
TABLE 4

TWENTY MOST IMPORTANT KNOWLEDGE BASES AND YES RESPONSES FOR WHERE BEST ACQUIRED

\begin{tabular}{|c|c|c|c|c|c|c|c|}
\hline \multirow[b]{2}{*}{ Knowledge Base } & \multirow[b]{2}{*}{$\begin{array}{l}\text { Library } \\
\text { School }\end{array}$} & \multirow[b]{2}{*}{$\begin{array}{c}\text { Other } \\
\text { Degree } \\
\text { Programs }\end{array}$} & \multirow[b]{2}{*}{ Internship } & \multicolumn{2}{|c|}{ Knowledge Source } & \multirow[b]{2}{*}{ On-the-Job } & \multirow[b]{2}{*}{ Other } \\
\hline & & & & $\begin{array}{l}\text { Continuing } \\
\text { Education } \\
\end{array}$ & $\begin{array}{c}\text { Staff } \\
\text { Development }\end{array}$ & & \\
\hline Bibliographic tools & 303 & 43 & 93 & 130 & 88 & 282 & 28 \\
\hline Percent & 31.3 & 4.4 & 9.6 & 13.4 & 9.1 & 29.2 & 2.9 \\
\hline Oral communication skills & 137 & 176 & 45 & 138 & 145 & 155 & 115 \\
\hline Percent & 15.0 & 19.3 & 4.9 & 15.2 & 15.9 & 17.0 & 12.6 \\
\hline Writing skills & 114 & 228 & 26 & 127 & 110 & 126 & 107 \\
\hline Percent & 13.6 & 27.2 & 3.1 & 15.2 & 13.1 & 15.0 & 12.8 \\
\hline Specialized reference sources & 248 & 61 & 84 & 132 & 93 & 276 & 21 \\
\hline Percent & 27.1 & 6.7 & 9.2 & 14.4 & 10.2 & 30.2 & 2.3 \\
\hline Decision making & 200 & 74 & 64 & 145 & 128 & 234 & 54 \\
\hline Percent & 22.3 & 8.2 & 7.1 & 16.1 & 14.2 & 26.0 & 6.0 \\
\hline Search strategy & 268 & 13 & 68 & 115 & 101 & 246 & 18 \\
\hline Percent & 32.3 & 1.6 & 8.2 & 13.9 & 12.2 & 29.7 & 2.2 \\
\hline Subject field & 73 & 239 & 39 & 136 & 40 & 157 & 51 \\
\hline Percent & 9.9 & 32.5 & 5.3 & 18.5 & 5.4 & 21.4 & 6.9 \\
\hline General reference sources & 308 & 22 & 93 & 112 & 91 & 267 & 21 \\
\hline Percent & 33.7 & 2.4 & 10.2 & 12.3 & 10.0 & 29.2 & \\
\hline Planning & 230 & 71 & 63 & 156 & 134 & 243 & 38 \\
\hline Percent & 24.6 & 7.6 & 6.7 & 16.7 & 14.3 & 26.0 & 4.1 \\
\hline Online searching & 263 & 4 & 66 & 160 & 116 & 242 & 15 \\
\hline Percent & 30.4 & 0.5 & 7.6 & 18.5 & 13.4 & 27.9 & 1.7 \\
\hline Reference interview & 259 & 5 & 81 & 80 & 82 & 232 & 13 \\
\hline Percent & 34.4 & 0.7 & 10.8 & 10.6 & 10.9 & 30.9 & 1.7 \\
\hline Selection of materials & 245 & 24 & 70 & 91 & 71 & 245 & 14 \\
\hline Percent & 32.2 & 3.2 & 9.2 & 12.0 & 9.3 & 32.2 & 1.8 \\
\hline Catalog codes/rules & 294 & 1 & 59 & 94 & 82 & 230 & 13 \\
\hline Perce & 38.0 & 0.1 & 7.6 & 12.2 & 10.6 & 29.8 & 1.7 \\
\hline Personnel management & 205 & 75 & 50 & 167 & 150 & 223 & 36 \\
\hline Perc & 22.6 & 8.3 & 5.5 & 18.4 & 16.6 & 24.6 & 4.0 \\
\hline Subject cataloging & 271 & 10 & 60 & 72 & 64 & 216 & 15 \\
\hline Perc & 38.3 & 1.4 & 8.5 & 10.2 & 9.0 & 30.5 & \\
\hline Library automation & 288 & 34 & 62 & 180 & 143 & 239 & 32 \\
\hline Percent & 29.5 & 3.5 & 6.3 & 18.4 & 14.6 & 24.4 & 3.3 \\
\hline Subject classification & 274 & 10 & 63 & 85 & 63 & 218 & 13 \\
\hline Percent & 37.7 & 1.4 & 8.7 & 11.7 & 8.7 & 30.0 & 1.8 \\
\hline Structure of subject literature & 236 & 84 & 47 & 97 & 47 & 197 & 17 \\
\hline Percent & 32.6 & 11.6 & 6.5 & 13.4 & 6.5 & 27.2 & 2.3 \\
\hline Bibliographic/library instruction & 241 & 15 & 66 & 131 & 118 & 233 & 9 \\
\hline Percent & 29.6 & 1.8 & 8.1 & 16.1 & 14.5 & 28.7 & 1.1 \\
\hline Staff training and development & 169 & 56 & 35 & 161 & & 222 & 29 \\
\hline Percent & 20.2 & 6.7 & 4.2 & 19.3 & 19.5 & 26.6 & 3.5 \\
\hline Totals & 4,626 & 1,245 & 1,234 & 2,509 & 2,029 & 4,483 & 659 \\
\hline Percent & 27.6 & 7.4 & 7.4 & 14.9 & 12.1 & 26.7 & 3.9 \\
\hline
\end{tabular}

"the library directors who constituted the respondent population generally declared themselves willing to consider the transfer of certain topics or issues from what might otherwise be an overburdened and cluttered curriculum to on-the-job training. However, when they were asked to suggest specific courses or topics amenable to such treatment, very few recommendations emerged, and no consensus was apparent."'

Another significant difference between the responses for where proficiencies were acquired and perceptions of where they are best acquired was that nine knowledge bases were perceived as best gained in continuing education and staff development activities. No skills were reported as having been most frequently acquired from these two sources, although they represented about 16 percent of the "yes" responses (see table 2). The participants recommended that the remaining proficiency, knowledge of a subject field, be obtained through another degree program. 
TABLE 5

TWENTY TOP KNOWLEDGE BASES RANKED BY AMOUNT HELD AND YES RESPONSES FOR WHERE BEST ACQUIRED

\begin{tabular}{|c|c|c|c|c|c|c|c|}
\hline \multirow[b]{2}{*}{ Knowledge Base } & \multirow[b]{2}{*}{$\begin{array}{l}\text { Library } \\
\text { School }\end{array}$} & \multirow[b]{2}{*}{$\begin{array}{l}\text { Other } \\
\text { Degree } \\
\text { Programs }\end{array}$} & \multirow[b]{2}{*}{ Internship } & \multicolumn{2}{|c|}{ Knowledge Source } & \multirow[b]{2}{*}{ On-the-Job } & \multirow[b]{2}{*}{ Other } \\
\hline & & & & $\begin{array}{l}\text { Continuing } \\
\text { Education }\end{array}$ & $\begin{array}{c}\text { Staff } \\
\text { Development }\end{array}$ & & \\
\hline Bibliographic tools & 303 & 43 & 93 & 130 & 88 & 282 & 28 \\
\hline Percent & 31.3 & 4.4 & 9.6 & 13.4 & 9.1 & 29.2 & 2.9 \\
\hline Writing skills & 114 & 228 & 26 & 127 & 110 & 126 & 107 \\
\hline Percent & 13.6 & 27.2 & 3.1 & 15.2 & 13.1 & 15.0 & 12.8 \\
\hline Search strategy & 268 & 13 & 68 & 115 & 101 & 246 & 18 \\
\hline Percent & 32.3 & 1.6 & 8.2 & 13.9 & 12.2 & 29.7 & 2.2 \\
\hline Specialized reference sources & 248 & 61 & 84 & 132 & 93 & 276 & 21 \\
\hline Percent & 7.1 & 6.7 & 9.2 & 14.4 & 10.2 & 30.2 & 2.3 \\
\hline Subject field & 73 & 239 & 39 & 136 & 40 & 157 & 51 \\
\hline Percent & 9.9 & 32.5 & 5.3 & 18.5 & 5.4 & 21.4 & 6.9 \\
\hline General reference sources & 308 & 22 & 93 & 112 & 91 & 267 & 21 \\
\hline Percent & 33.7 & 2.4 & 10.2 & 12.3 & 10.0 & 29.2 & 2.3 \\
\hline Reference interview & 259 & 5 & 81 & 80 & 82 & 232 & 13 \\
\hline Percent & 34.4 & 0.7 & 10.8 & 10.6 & 10.9 & 30.9 & 1.7 \\
\hline Oral communication skills & 137 & 176 & 45 & 138 & 145 & 155 & 115 \\
\hline Percent & 15.0 & 19.3 & 4.9 & 15.2 & 15.9 & 17.0 & 12.6 \\
\hline Online searching & 263 & 4 & 66 & 160 & 116 & 242 & 15 \\
\hline Percent & 30.4 & 0.5 & 7.6 & 18.5 & 13.4 & 27.9 & 1.7 \\
\hline Selection of materials & 245 & 24 & 70 & 91 & 71 & 245 & 14 \\
\hline Percent & 32.2 & 3.2 & 9.2 & 12.0 & 9.3 & 32.3 & 1.8 \\
\hline Filing & 231 & 4 & 47 & 43 & 65 & 229 & 21 \\
\hline Percent & 36.1 & 0.6 & 7.3 & 6.7 & 10.2 & 35.8 & 3.3 \\
\hline Subject classification & 274 & 10 & 63 & 85 & 63 & 218 & 13 \\
\hline Percent & 37.7 & 1.4 & 8.7 & 11.7 & 8.7 & 30.0 & 1.8 \\
\hline Catalog codes/rules & 294 & 1 & 59 & 94 & 82 & 230 & 13 \\
\hline Percent & 38.0 & 0.1 & 7.6 & 12.2 & 10.6 & 29.8 & 1.7 \\
\hline Research methods & 244 & 177 & 37 & 124 & 67 & 117 & 39 \\
\hline Percent & 30.3 & 22.0 & 4.6 & 15.4 & 8.3 & 14.5 & 4.8 \\
\hline Bibliographic/library instruction & 241 & 15 & 66 & 131 & 118 & 233 & 9 \\
\hline Percent & 29.6 & 1.8 & 8.1 & 16.1 & 14.5 & 28.7 & 1.1 \\
\hline Structure of subject literature & 236 & 84 & 47 & 97 & 47 & 197 & 17 \\
\hline Percent & 32.6 & 11.6 & 6.5 & 13.4 & 6.5 & 27.2 & 2.3 \\
\hline Subject cataloging & 271 & 10 & 60 & 72 & 64 & 216 & 15 \\
\hline Percent & 38.3 & 1.4 & 8.5 & 10.2 & 9.0 & 30.5 & 2.1 \\
\hline Decision making & 200 & 74 & 64 & 145 & & 234 & 54 \\
\hline Percent & 22.3 & 8.2 & 7.1 & 16.1 & 14.2 & 26.0 & 6.0 \\
\hline Higher education & 121 & 103 & 20 & 77 & 43 & 145 & 62 \\
\hline Percent & 21.2 & 18.0 & 3.5 & 13.5 & 7.5 & 25.4 & 10.9 \\
\hline Planning & 230 & 71 & 63 & 156 & 134 & 243 & 38 \\
\hline Percent & 24.6 & 7.6 & 6.7 & 16.7 & 14.3 & 26.0 & 4.1 \\
\hline Totals & 4,560 & 1,364 & 1,191 & 2,245 & 1,748 & 4,290 & 684 \\
\hline Percent & 28.4 & 8.5 & 7.4 & 14.0 & 10.9 & 26.7 & 4.3 \\
\hline
\end{tabular}

Apparently, relatively new librarians and library directors are not in complete agreement on this issue. The librarians participating in this study supported continuing education as a valuable learning experience and suggested skills best learned through such programs. White and Paris observed that library directors supported continuing education but "there would appear to be no consensus that anything in particular should be ... acquired through continuing education." 10

The tables also reflect the respondents' shift from on-the-job training (see table 2) to continuing education and staff development (see table 4). While the total percentages of "yes" responses remained about the same for library school as a source of knowledge, total "yes" responses for "on the job" dropped from 35 percent to about 27 percent and continuing education and 
TABLE 6

SUMMARY OF YES RESPONSES TO WHERE KNOWLEDGE WAS ACQUIRED AND WHERE IT IS BEST ACQUIRED

\begin{tabular}{|c|c|c|c|c|c|c|c|}
\hline \multirow[b]{2}{*}{ Knowledge Base } & \multirow[b]{2}{*}{$\begin{array}{l}\text { Library } \\
\text { School } \\
\end{array}$} & \multirow[b]{2}{*}{$\begin{array}{c}\text { Other } \\
\text { Degree } \\
\text { Programs }\end{array}$} & \multirow[b]{2}{*}{ Internship } & \multicolumn{2}{|c|}{ Knowledge Source } & \multirow[b]{2}{*}{ ntOn-the-Job } & \multirow[b]{2}{*}{ Other } \\
\hline & & & & $\begin{array}{l}\text { Continuing } \\
\text { Education }\end{array}$ & $\begin{array}{c}\text { Staff } \\
\text { Developmen }\end{array}$ & & \\
\hline Most important and & 3,881 & 1,055 & $\begin{array}{l}539 \\
4.0 \%\end{array}$ & 1,290 & 929 & 4,750 & 1,105 \\
\hline Most important and & 4,626 & 1,245 & 1,234 & 2,509 & 2,029 & 4,483 & 659 \\
\hline where best acquired & $27.6 \%$ & $7.4 \%$ & $7.4 \%$ & $14.9 \%$ & $12.1 \%$ & $26.7 \%$ & 3.9 \\
\hline Change in percentage & $-1.0 \%$ & $-0.4 \%$ & $+3.4 \%$ & $+5.4 \%$ & $+5.2 \%$ & $-8.4 \%$ & $-4.3^{\circ}$ \\
\hline Amount held and & 3,938 & 1,281 & 550 & 1,161 & 746 & 4,543 & 1,122 \\
\hline where acquired & $29.5 \%$ & $9.6 \%$ & $4.1 \%$ & $8.7 \%$ & $5.6 \%$ & $34.1 \%$ & $8.4 \%$ \\
\hline Amount held and & 4,560 & 1,364 & 1,191 & 2,245 & 1,748 & 4,290 & 684 \\
\hline where best acquired & $28.4 \%$ & $8.5 \%$ & $7.4 \%$ & $14.0 \%$ & $10.9 \%$ & $26.7 \%$ & $4.3 \%$ \\
\hline Change in percentage & $-1.1 \%$ & $-1.1 \%$ & $+3.3 \%$ & $+5.3 \%$ & $+5.3 \%$ & $-7.4 \%$ & $\quad-4.1 \%$ \\
\hline
\end{tabular}

staff development combined jumped from 16 percent to 27 percent. Stated another way, all but two of the ten skills that dropped out of on-the-job training as an actual source of information ended up in the continuing education/staff development category as a recommended source of job skills.

An analysis of the twenty top knowledge bases as ranked by the perception of the amount of knowledge held (see table 5) revealed few differences. Again, the use of different rankings produced only three differences: research methods, filing, and higher education replaced personnel management, library automation, and staff training and development. Filing did shift from the on-the-job training category (see table 3 ) to the library school category (see table 5). The total percentages of "yes" responses are about the same in tables 4 and 5 . A comparison of totals in tables 3 and 5, however, reveal changes in percentages similar to those evidenced by tables 2 and 4 . That is, internships, continuing education, and staff development activities were more often reported as preferred than as actual sources of knowledge while the reverse was true for on-the-job training. Responses for library school again remained about the same.

Table 6, which presents only the totals from tables 2 through 5 , further illustrates the differences just discussed. Comparing the "where acquired" and "where best acquired" cells in the upper and lower halves of the table, the major increases are in the continuing education and staff development columns, and the major decreases are in the on-the-job column. The increases in the percentages for the internship column are significant as well.

\section{SUMMARY AND CONCLUSIONS}

This article has focused on the second phase of a study of ARL librarians and their perceptions of the amount and importance of their professional knowledge, where they acquired such knowledge, and where they think it should be acquired. Findings of the first phase indicated that, while a traditional core of library knowledge is still highly valued, proficiencies in areas such as automation and management are deemed important by this group of librarians as well. ${ }^{11}$ Of concern, however, is the fact that these librarians seem to lack substantial knowledge in some of the areas that they perceive as important. Assuming that is indeed the case, it becomes critical to identify where librarians are acquiring what knowledge they do have and to develop recommendations for where relevant knowledge can be obtained most effectively. Such concerns provided the major rationale for the additional data analysis in this report.

The second stage of the data analysis shed light on where librarians gain their 
knowledge. Formal library school programs and on-the-job training account for the bulk of the professional knowledge among the study's participants, with library schools imparting more traditional learning. Study participants prefer to rely more heavily on continuing education and staff development programs and less heavily on on-the-job experience. Their recommendations for what should be learned in library school remain about the same as reported in the first analysis.

A shortage of appropriate opportunities may be the reason why librarians have been learning more on the job than through continuing education. As White and Paris noted, "reports from professional societies, state agencies, and individual libraries report a level of participation [in continuing education] which touches at best only a small part of the pro- fession and then only haphazardly." ${ }^{12}$ Yet academic librarians, at least, appear to prefer continuing education over on-thejob training as a mode of learning.

If librarians are not content to acquire as much of their professional expertise on the job as in the past, then appropriate agencies may need to expand their continuing education offerings and library administrators may need to strengthen their staff development programs and support for alternative opportunities. Schools of library and information science may be well advised to take another look at the desirability of expanding their curricula to twoyear programs, as some have done already. In short, an appropriate variety of educational opportunities will be necessary to meet the increasing needs of librarians in a more complex environment.

\section{REFERENCES AND NOTES}

1. Millicent D. Abell, "The Changing Role of the Academic Librarian: Drift and Mastery," College \& Research Libraries 40:154-64 (Mar. 1979).

2. Toni Carbo Bearman, "The Changing Role of the Information Professional," Library Trends 32: 255-60 (Winter 1984).

3. Patricia Battin, "Developing University and Research Library Professionals: A Director's Perspective," American Libraries 14: 22-25 (Jan. 1983).

4. Sheila Creth and Faith Harders, "Requirements for the Entry Level Librarian," Library Journal 105: 2168-69 (Oct. 1980).

5. Association of Research Libraries, Education for the Research Library Professional, minutes of the 97th meeting, Oct. 1980, Arlington, Virginia (Washington, D.C.: ARL, 1981), p.28-35. See also Maurice P. Marchant and Nathan M. Smith, "The Research Library Director's View of Library Education," College \& Research Libraries 43: 437-44 (Nov. 1982).

6. Jose-Marie Griffiths and Donald W. King, New Directions in Library and Information Science Education (White Plains, N.Y.: Knowledge Industry, 1986).

7. Ronald R. Powell and Sheila D. Creth, "Knowledge Bases and Library Education," College \& Research Libraries 47: 16-27 (Jan. 1986).

8. A recent study found that the directors of large academic libraries recommended sixteen courses deemed essential for the preparation of entry-level professionals. Of those sixteen courses, ten were primarily bibliography or reference courses, two dealt with collection development, two with cataloging and technical services, one with management, and one with automation. See Herbert S. White and Marion Paris, "Employer Preferences and the Library Education Curriculum," Library Quarterly, 55:1-33 (Jan. 1985).

9. Ibid., p.29.

10. Ibid., p.14.

11. Powell and Creth, "Knowledge Bases," p.25.

12. White and Paris, "Employer Preferences," p.30. 